\title{
Computer-assisted, template-guided immediate implant placement and loading in the mandible: a case report
}

Thomas Spielau ${ }^{1 *}$, Uli Hauschild ${ }^{2}$ and Joannis Katsoulis ${ }^{3}$

\begin{abstract}
Background: Computer-assisted implant planning has become an important diagnostic and therapeutic tool in modern dentistry. This case report emphasizes the possibilities in modern implantology combining virtual implant planning, guided surgery with tooth and implant supported templates, immediate implant placement and loading.

Case presentation: A straight forward approach was followed for the mandible presenting with hopeless lower incisors. Diagnosis, decision making and treatment approach were based on clinical findings and detailed virtual threedimensional implant planning. Extractions of the hopeless mandibular incisors, immediate and guided implant placement of six standard implants, and immediate loading with a provisional fixed dental prosthesis (FDP) were performed fulfilling patient's functional and esthetic demands. The final computer assisted design / computer assisted manufacturing (CAD/ CAM) FDP with a titanium framework and composite veneering was delivered after 6 months. At the 1 -year recall the FDP was free of technical complications. Stable bony conditions and a healthy peri-implant mucosa could be observed.

Conclusions: Computer assisted implantology including three-dimensional virtual implant planning, guided surgery, and CAD/CAM fabrication of provisional and final reconstructions allowed for a concise treatment workflow with predictable esthetic and functional outcomes in this mandibular full-arch case. The combination of immediate implant placement and immediate loading was considerably more complex and required a high level of organization between implantologist, technician and patient. After the usage of a first tooth-supported surgical template with subsequent extraction of the supporting teeth, a second surgical template stabilized on the previously inserted implants helped to transfer the planned implant position in the extraction sites with a guided approach.
\end{abstract}

Keywords: Virtual implant planning, Guided surgery, Immediate placement, Immediate loading, CAD/CAM

\section{Background}

Computer assisted implantology (CAI) was introduced more than 25 years ago and aimed to facilitate implant planning and to avoid intraoperative complications such as mandibular nerve damage, sinus perforations, fenestrations, or dehiscence [1-4]. Based on a computerized tomography (CT) scan and a digitized tooth setup, the prosthetically ideal implant positions can be planned virtually with the help of a guided surgery software allowing for three dimensional visualization prior to implant surgery $[2,5,6]$.

\footnotetext{
* Correspondence: info@ergozahn.de

${ }^{1}$ Implantology, Oral Surgery; Private dental office, Johannesstrasse 7-9,

474623 Kevelaer, Germany

Full list of author information is available at the end of the article
}

Furthermore, the possibility to transfer the virtually planned implant position to the real clinical situation is provided by a stereolithographically fabricated surgical template [3, 7]. While only few guided implant placement systems were available at the time, today, multiple CAI software are available on the market. Several in-vitro, cadaver and clinical studies have reported on the accuracy of guided implant placement [8-10]. Although the current state of software and hardware technology has improved, inaccuracies in implant placement may occur and depend on different factors such as the template support (bone, mucosa, teeth, implants), intrinsic factors of the surgical guide (tolerance in diameter between the drill and the guide sleeve, fabrication 


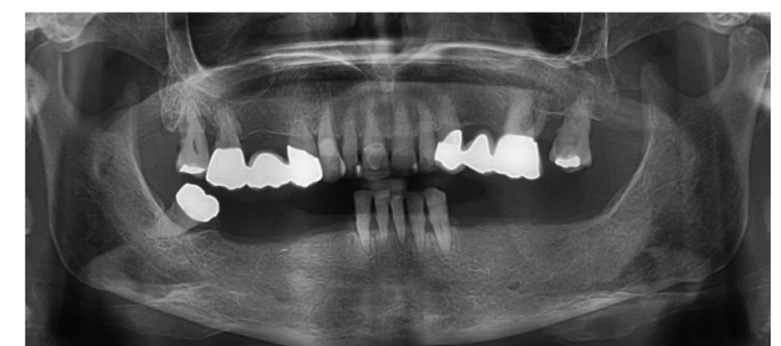

Fig. 1 Panoramic radiograph of the initial dental status

accuracy of the guide) [11, 12] and human related factors during the workflow of virtual planning and guided surgery $[7,13]$. The guided surgery approach is still controversially discussed [14-16] even though the procedure may be performed in a safe and predictable way $[17,18]$. However, a systematic and concise approach performing the single steps in the treatment sequence may allow for more accurate implant positioning as type of guide and fixation have an important influence $[19,20]$. Additionally, the use of multiple templates with different supports, i.e. teeth and implant support combined in a sequenced order is believed to improve accuracy compared to a mucosa supported approach alone [21].

While some patients wish to be informed in detail about the specific treatment steps, most of them want to know whether they would have to leave the dental office without teeth at some point of the treatment. In this context, immediate implant placement after tooth extraction and immediate implant loading with a fixed provisional reconstruction may help the patient as time after extractions and osseointegration is consolidated. In guided surgery protocols, minimally invasive placement and immediate loading has been a possible treatment step from the beginning [3, 4]. Postoperative morbidity after flapless surgery is significantly reduced compared to the traditional open approach, especially in edentulous patients $[17,22,23]$. Later during the treatment, reconstructions fabricated with the help of computer assisted design / computer assisted manufacturing (CAD/CAM) provide high quality and aesthetic materials. Although $\mathrm{CAI}$ and CAD/CAM procedures have facilitated towards a straight forward workflow in the rehabilitation of edentulous patients, immediate implant placement and immediate loading protocols combined are complex and required a high level of organization between the implantologist, the technician and the patient.

The aim of the present case report was to illustrate the feasibility of combined immediate implant placement and loading approach using CAI in the rehabilitation of a patient with a partially dentate mandible asking for a comprehensive treatment and, specifically, not accepting being edentulous all the while.

\section{Case report}

Initial status und treatment concept

The partially dentate 74-year old patient presented with masticatory problems due a removable partial denture (RPD) with insufficient stability in combination with chronic pain condition in the lower front teeth area. She asked for a comprehensive treatment and did not accept to be edentulous at any stage of the treatment. The patient was a non-smoker and -with the help of antihypertensive (Candecor comp. $32 \mathrm{mg} / 12,5 \mathrm{mg}$ ) and anticoagulant medication (quick 30; Marcourmar)- in good general health.

The dental status showed an acceptable oral hygiene, some teeth with increased mobility grade III $(41 / 31 / 32$ and 18,28$)$ and local periodontal problems including horizontal bone loss $(42 / 41 / 31 / 32 / 33$, 18/17, 27/28). The teeth 42 and 33 were healthy and not mobile. The alveolar crest in the lateral

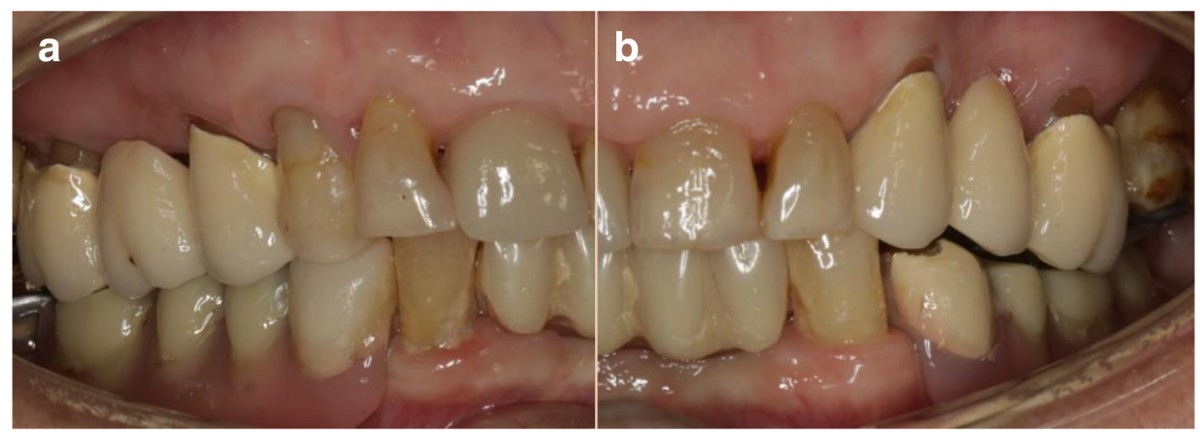

Fig. 2 Initial dental status; a right side; b left side 

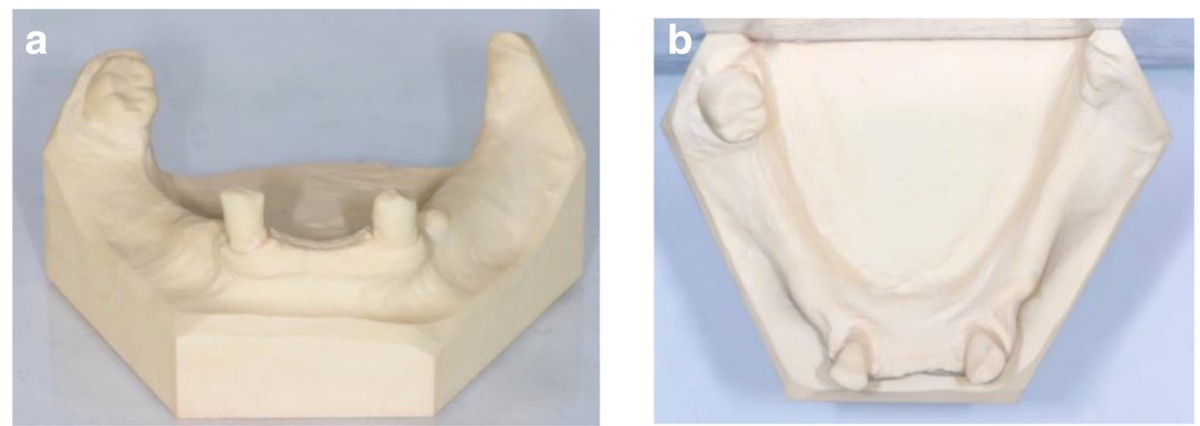

Fig. 3 Occlusal and frontal view of the study models after extraction of the 3 incisors 41,31 and 32; a front view, b occlusal view

mandible area showed clinically a wide shape with thick keratinized mucosa. The initial panoramic radiograph revealed stable crestal bone in the lateral mandible area (Figs. 1, 2 and 3). Thus, focusing on the lower jaw, the single tooth prognosis was fair for the teeth 47, 42 and 33 and hopeless for the teeth $41 / 31 / 32$ [24].

During decision making for the final treatment plan different options were discussed with the patient. Various treatment options including a removable dental prosthesis were discussed with the patient. To keep the patient's wish for a fixed reconstruction and to never become edentulous in any treatment phase and considering the prognosis of the remaining mandibular teeth, the decision was made to prepare a provisional fixed prosthesis with an immediate loading approach extracting the teeth 42 and 33 for prosthodontic reasons but maintaining tooth 47.

\section{Digital implant planning (Table 1)}

After extraction of the painful and extremely mobile lower front teeth $41 / 31 / 32$ and adaptation of the existing RPD, a cone beam computed tomography (CBCT) (Pax-Uni 3D, orangedental GmbH \& Co. KG, Biberach, Germany) with a $5 \times 8 \mathrm{~cm}$ field of view and $85 \mathrm{KV} / 5.5 \mathrm{~mA} / 0.2 \mathrm{~mm}$ Voxel was performed to proceed with the detailed implant planning (Fig. 4). Based on the anatomical conditions and prosthetic planning (i.e. tooth setup for the provisional RPD), six implants were virtually planned (3Diagnosys, 3DIEMME, Cantu, Italy) in the FDI (Fédération Dentaire Internationale) positions 46, 44, 42, 33, 35, and 36. As the implant positions 42 and 33 interfered with the teeth 43 and 33, a two-step procedure with two surgical templates was planned for the guided implant placement (Fig. 5a, b). The templates were fabricated stereolithographically (DS3000, XFAB, DWS srl, Thiene, Italy) according to the virtual implant planning. Based on the same digital file (Fig. 6a, b) a provisional fixed dental prosthesis (FDP) was prepared preoperatively allowing for an intraoral adaptation between the abutments and the framework to achieve a passive fit (Fig. 7a-d).

\section{Immediate implant placement}

During the day of surgery, a single dose of antibiotic ( $2 \mathrm{~g}$ of amoxicillin and clavulanic acid) was administered prophylactically $1 \mathrm{~h}$ prior to surgery. This treatment continued for five days $(1 \mathrm{~g}$ amoxicillin and clavulanic acid twice a day). Prior to the start of

Table 1 Material and software used for the planning and realization of the treatment

\begin{tabular}{lll}
\hline CBCT & Pax-Uni 3D & Orangedental GmbH \& Co. KG, Biberach a. d. Riß, Germany \\
\hline Virtual implant planning & 3Diagnosys ${ }^{\oplus}$ & 3DIEMME, Cantu, Italy \\
Implants & Thommen ELEMENT RC $4.5 \times 9.5 \mathrm{~mm}$ & Thommen Medical AG, Grenchen, Switzerland \\
CAD & Exocad & Exocad Gmbh, Darmstadt, Deutschland \\
CAM & M1 Wet & Zirkonzahn, Gais, Italy \\
Provisional FDP & Prefabricated titanium abutments CAD/CAM & Thommen Medical AG, Grenchen, Switzerland \\
& CoCr framework Composite veneering \& teeth & Sintermetall, Zirkonzahn Srl, Gais, Italy SR Nexco \\
& & Paste, Ivoclar Vivadent AG, Schaan, Liechtenstein \\
Final FDP & CAD/CAM CoCr framework Composite & Sintermetall, Zirkonzahn Srl, Gais, Italy SR Nexco \\
& veneering \& teeth & Paste, Ivoclar Vivadent AG, Schaan, Liechtenstein \\
\hline
\end{tabular}




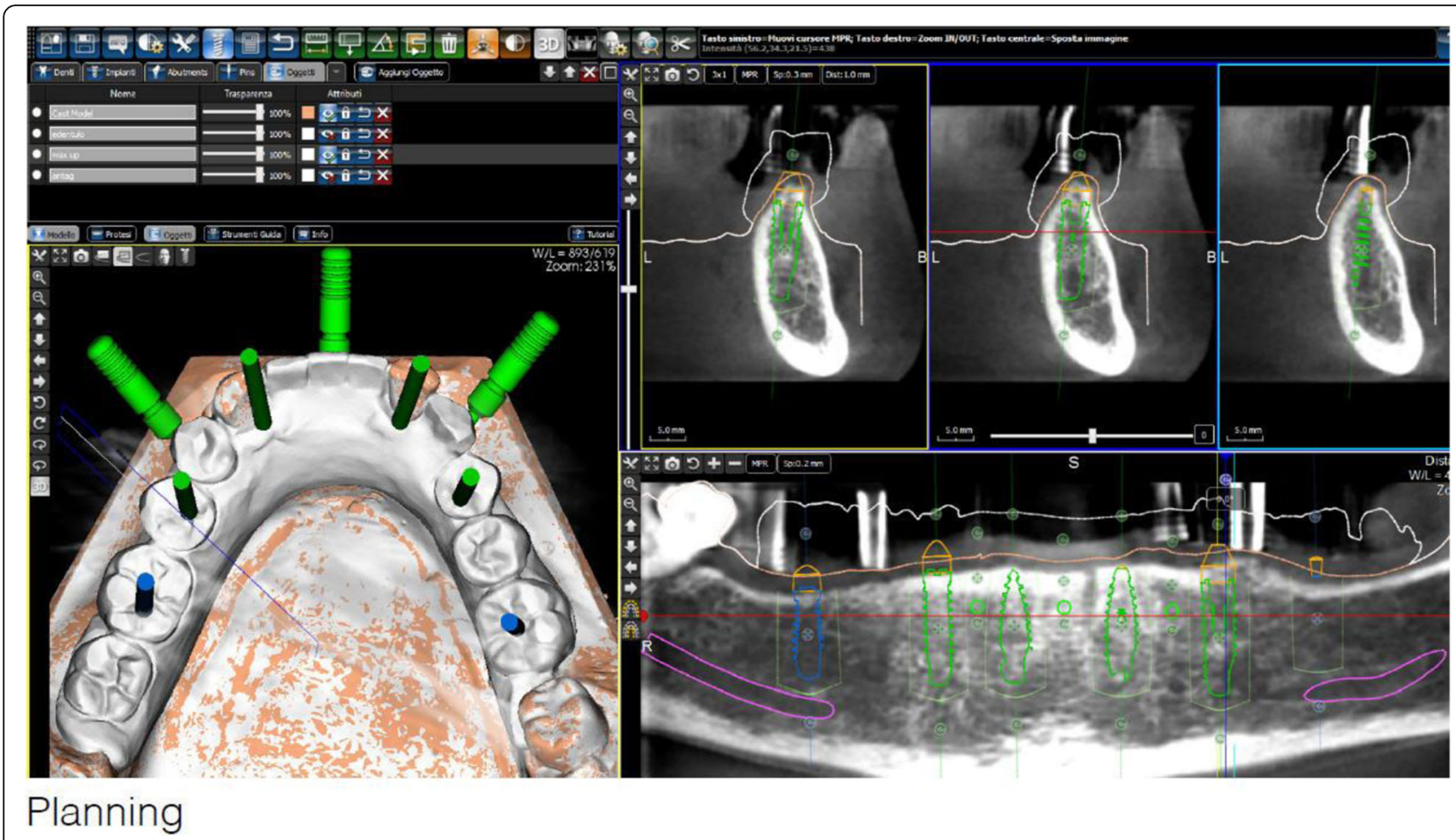

Fig. 4 Screen shot of the virtual implant planning (FDA) positions 36,35,33,42,and,46 occlusal, sectional and panoramic views
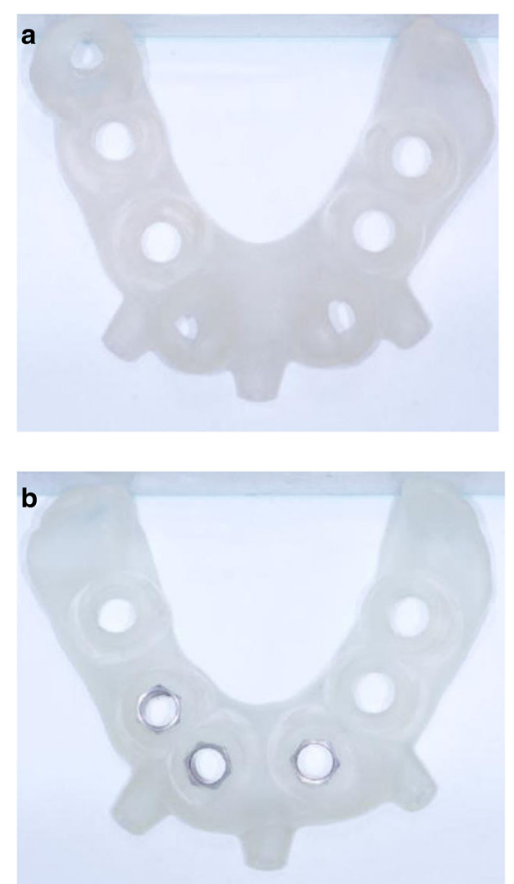

Fig. 5 CAD/CAM fabricated surgical guides no. 1 (a, tooth and mucosa supported) and no. 2 (b, implant and mucosa supported) surgery, the patient rinsed with $0.2 \%$ chlorhexidine for $1 \mathrm{~min}$. Local anesthesia was induced by using a $4 \%$ articaine solution with epinephrine 1:100.000.

The two-step approach comprised the flapless guided insertion of the four posterior implants (Thommen Element RC $4.5 \times 9.5 \mathrm{~mm}$, Thommen Medical AG, Grenchen, Switzerland), with the first surgical template that was tooth supported (Fig. 8a). The template was then removed and the teeth 42 and 33 previously supporting the guide were extracted. Thereafter, the second surgical template was positioned and stabilized on the four posterior implants with the help of specific abutments and the same anchor pins (Fig. 8b), thus allowing to place the anterior implant 42 and 33 (Thommen Element RC $4.5 \times 9.5$ $\mathrm{mm}$ ) guided and immediately after extractions. All the implants were inserted with a torque of $35 \mathrm{Ncm}$ and proved good primary stability.

\section{Immediate loading}

After removal of the second surgical template, the standard titanium abutments were mounted on the implants with a torque of $15 \mathrm{Ncm}$ (Fig. 9a). The gaps between the abutments and the FDP were filled with Dual-Composite material and the screw retained immediate provisional FDP delivered. The occlusion 

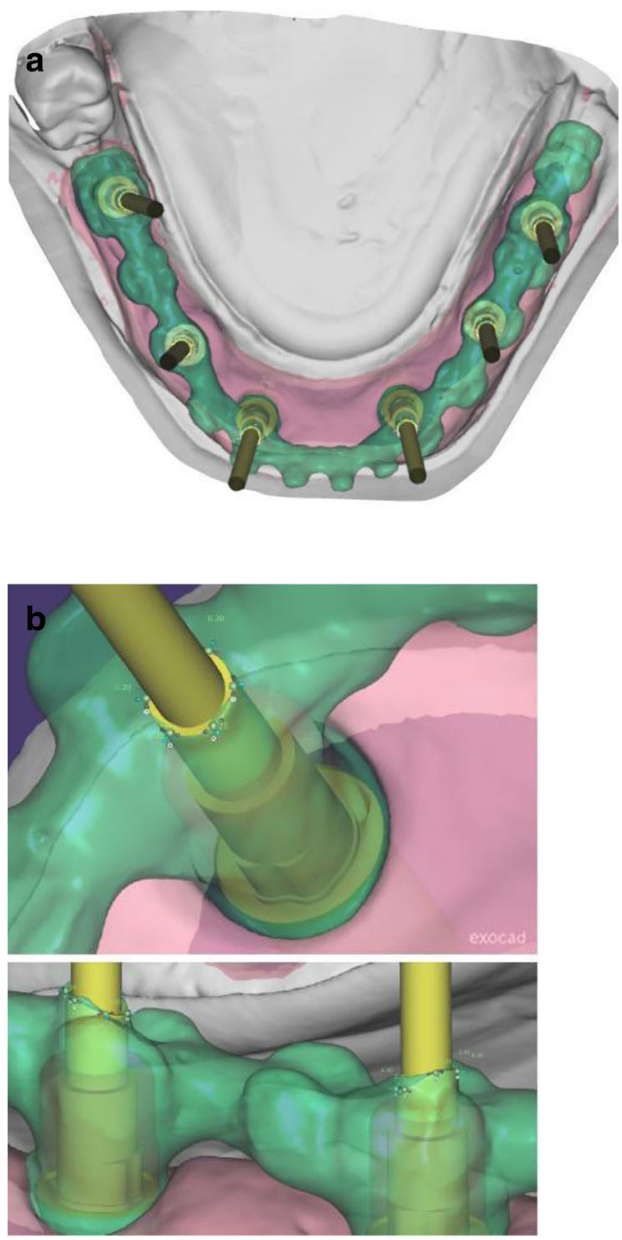

Fig. 6 Occlusal view showing CAD of the provisional FDP framework (a) and close-up view from the interface between the CAD framework and the prefabricated titanium abutments (b)

required only minor adaptations due to the accurate digital preoperative planning (Fig. 9b). The postoperative panoramic radiograph (OPT) showed the parallel axes of the six implants (Fig. 10).

\section{Final fixed prosthesis}

All the six implants osseointegrated successfully without complications. After 6 months with the provisional FDP a conventional impression was taken (screw retained impression copings, open tray technique, polyether material) to fabricate the final FDP on a new precise cast (Fig. 11), which was then digitized with a laboratory scanner (Deluxe Scanner, Open Technologies, Rezzato, Italy). The final framework was designed with straight connection to the implant platforms and with a cut-back allowing for the veneering material (Fig. 12a, b). While the cobalt-chromium framework was fabricated using
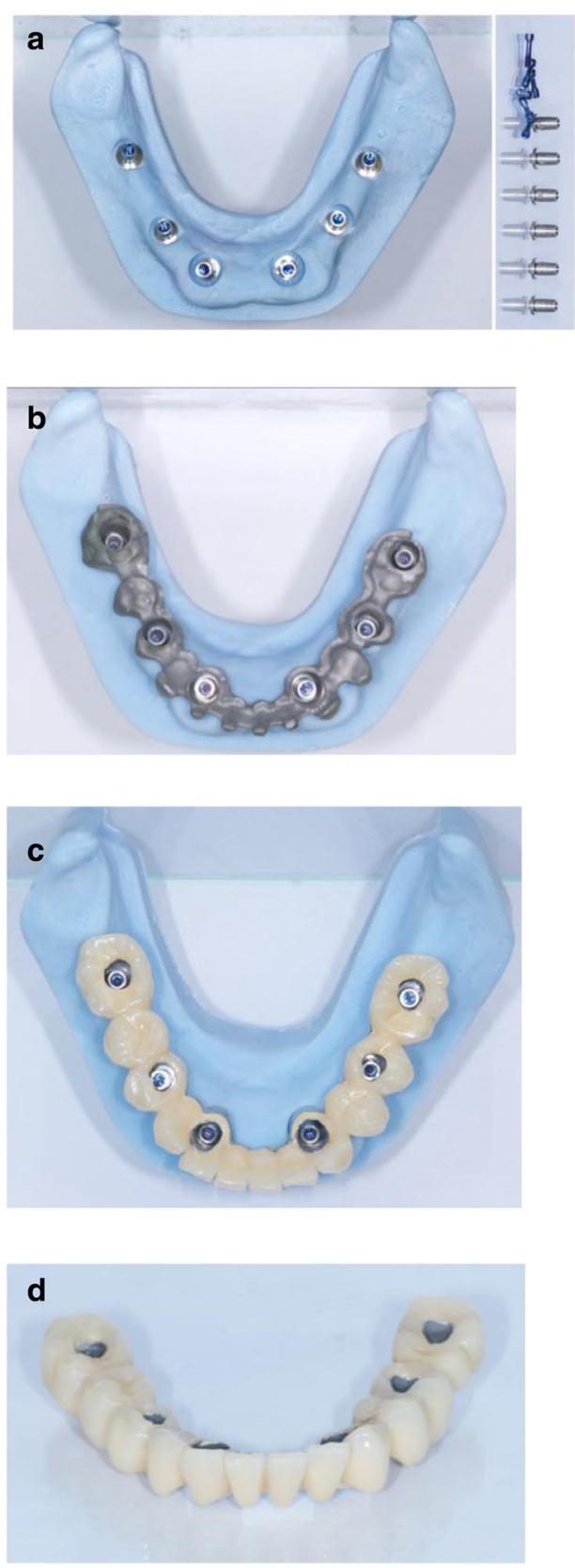

Fig. 7 CAD/CAM model with the prefabricated titanium abutments (a), the CAD/CAM cobalt-cromium framework (b), and the composite veneered provisional FDP $(\mathbf{c}, \mathbf{d})$ before bonding to the abutments

CAD/CAM technology (Exocad, Exocad gmbH, Deutschland / M1 Wet, Zirkonzahn, Italy) the veneering was performed manually allowing for an individual characterization of the teeth (Fig. 13a-d). The models were fabricated with a laser stereolithography printer (XFAB, DWS srl, Thiene, Italy) using an ABS-like polymer (RD096B, DWS srl, Thiene, 

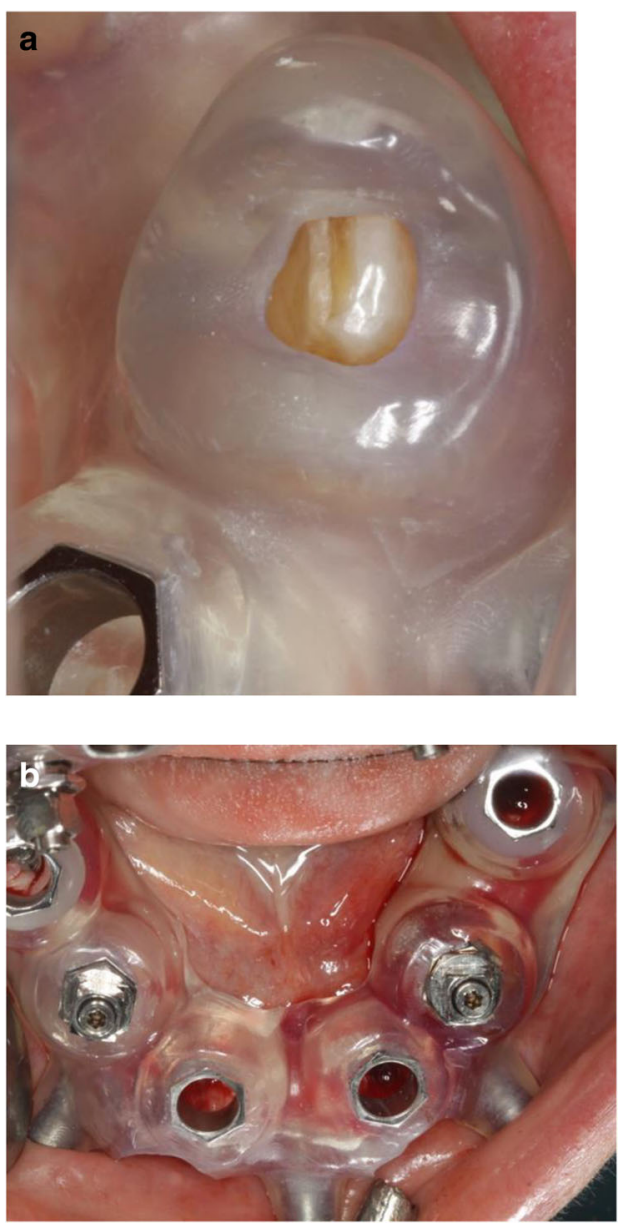

Fig. 8 Close-up view of the CAD/CAM guide no. 1 in-situ (tooth and mucosa supported) showing the perfect fit on tooth 33 (a), and occlusal view (b) of the CAD/CAM guide no. 2 (implant and mucosa supported) after extraction of the teeth $42 / 33$ and placement of the implants $44 / 35$

Italy). Healthy mucosal conditions were present at the delivery of the final CAD/CAM reconstruction made from cobalt-chromium and composite veneering material (Fig. 14a-f). The accurately fitting FDP was screw retained with $25 \mathrm{Ncm}$ and the screw access area covered with composite material. The OPT at the day of delivery showed optimal prosthetic and osseous conditions (Fig. 15). The patient followed a regular maintenance program at the dental hygienist twice a year.

At the one year follow-up appointment, healthy mucosal and stable crestal peri-implant conditions could be observed (Fig. 16). The patient was very pleased with the esthetic and functional outcome. Thus, the performed treatment was successful and showed stable results without complications or need for maintenance service after the first year.
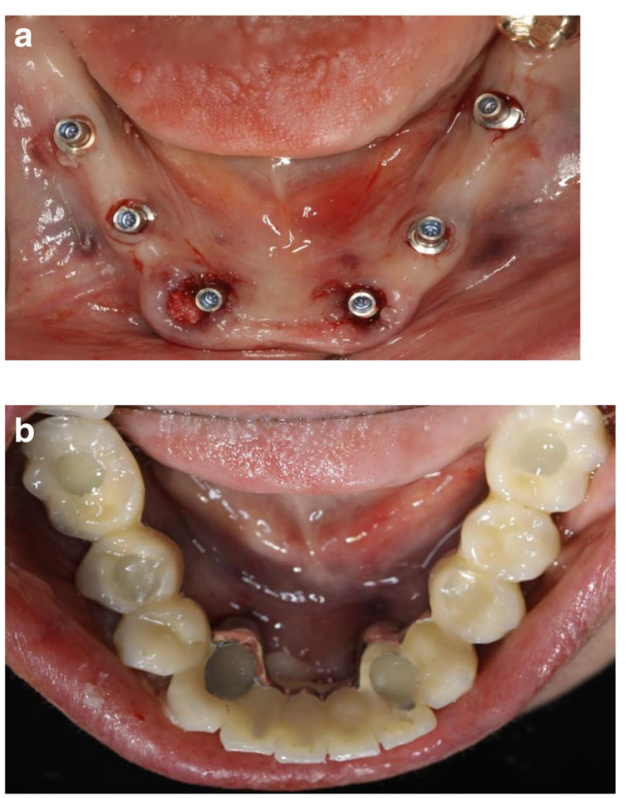

Fig. 9 a; Occlusal views of the abutments and $\mathbf{b}$; the immediate provisional reconstruction that were passively bond in-situ

\section{Discussion}

The use of CAI software in the preoperative virtual three-dimensional implant planning allowed for guided and immediate implant placement, and proved to be especially beneficial in the presented mandibular full-arch case. While there are some studies that investigated outcomes of immediately loaded implants placed in edentulous patients using computer-assisted template-guided surgery to support a FDP [25], only few case reports are available in literature describing the entire workflow, the patients state in detail, and the usage of guided surgery templates with subsequent immediate loading [3, 4]. The considerably more complex combination of immediate implant placement and immediate loading required a high level of organization between implantologist and technician, minimizing patient's compliance. Pozzi et al. reported excellent results with CAD/CAM cross-arch Zirconia bridges on

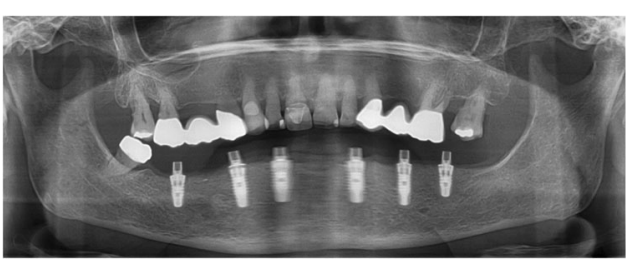

Fig. 10 Postoperative panoramic radiograph 


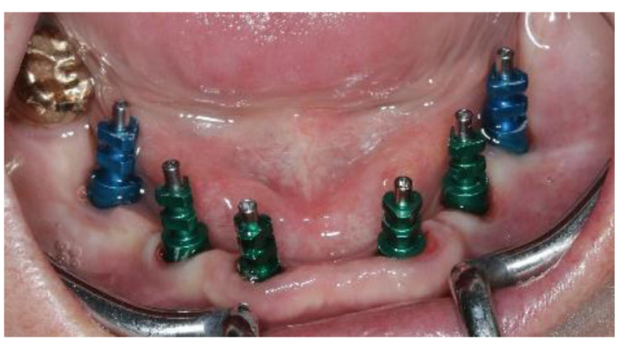

Fig. 11 Frontal view showing the screw retained post at impression taking 24 weeks after implant placement

immediately loaded implants placed with computerassisted/template-guided surgery [26]. Several investigators presented analyses of recent studies in this context elaborating the factors that influence mainly the accurate implant placement but also the comparable outcome of the reconstructions after guided implant placement $[15,20,21,27-31]$. In the present case report, two CAD/CAM surgical templates were combined in this partially dentate patient with extraction of the teeth $42 / 33$ and immediate implants performed in a sequenced order. The first scanner-based template was teeth and mucosa supported enabling a higher template stability and, thus,

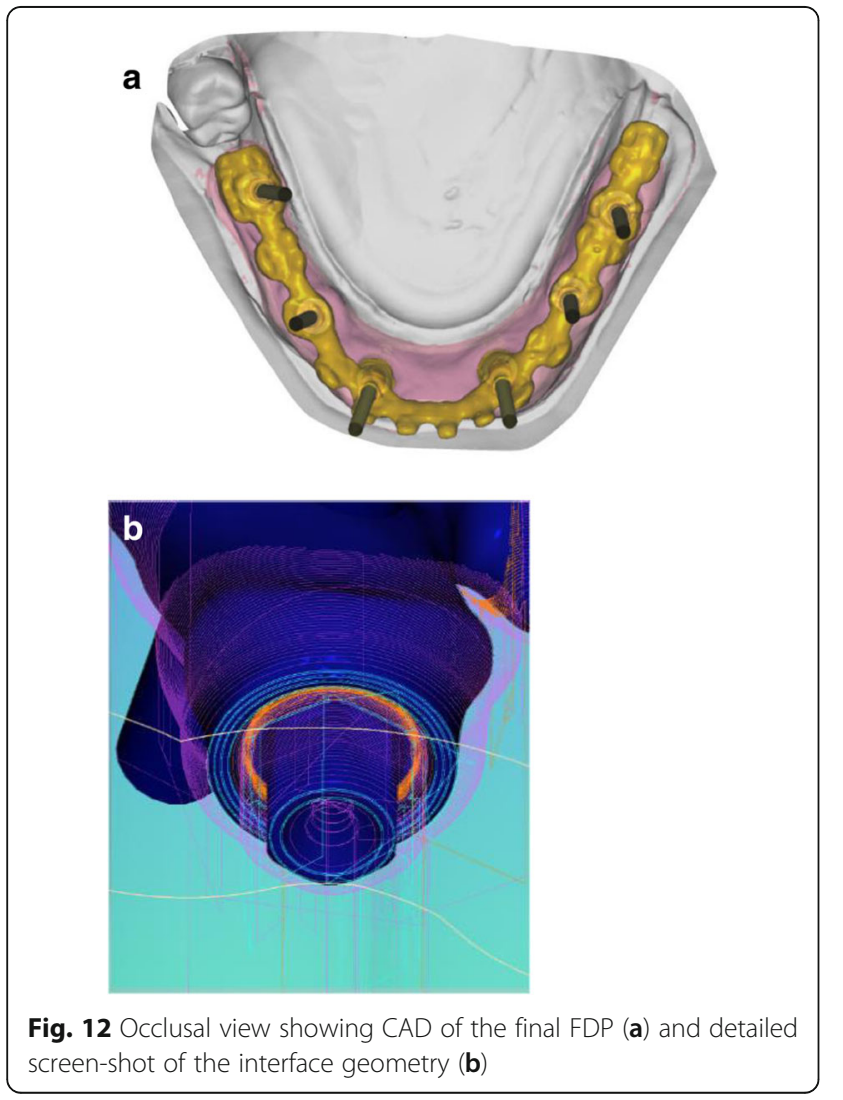

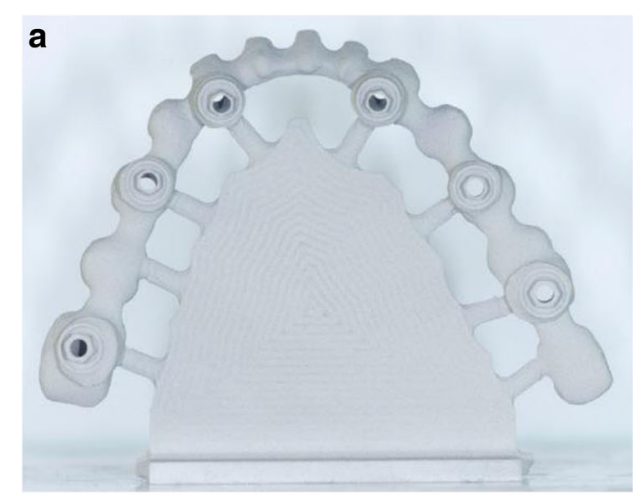
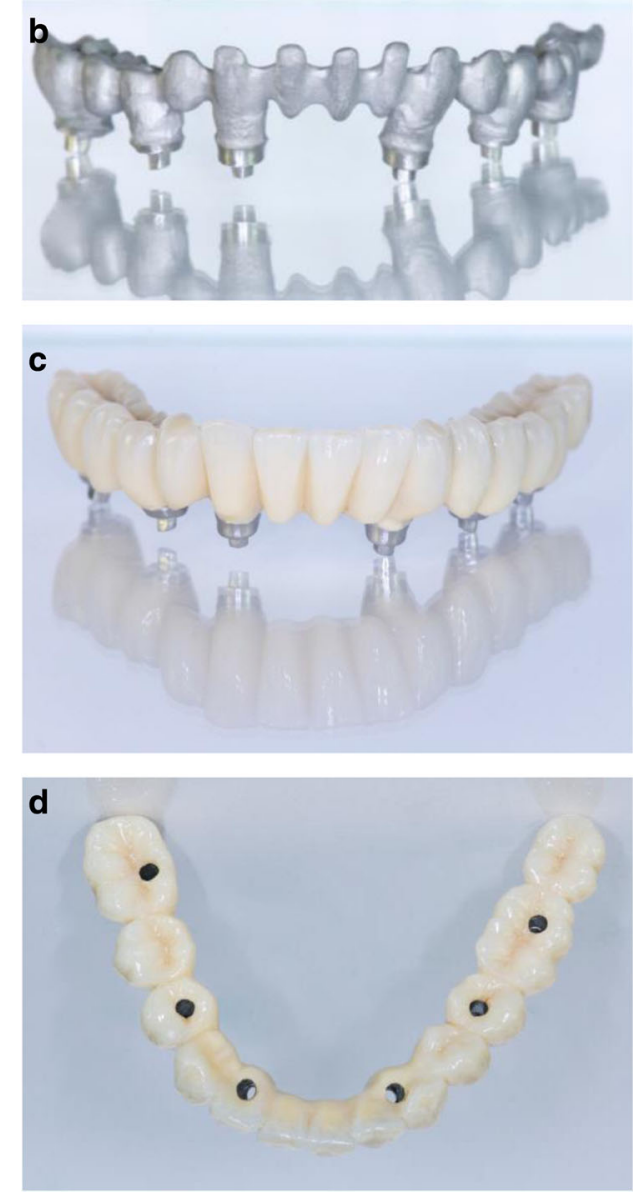

Fig. 13 CAD/CAM fabricated one-piece cobalt-cromium framework before $(\mathbf{a}, \mathbf{b})$ and after veneering with composite (Nexco Ivoclar) i.e. final $\operatorname{FDP}(\mathbf{c}, \mathbf{d})$

more accurate guided osteotomies and implant placement. Four posterior implants were placed with this approach allowing to support the second surgical template after extraction of the anterior teeth 42 / 33. The stability on these four points was high as the implants $42 / 33$ showed a torque value of 35 to $40 \mathrm{Ncm}$ each. The subsequent two anterior immediate implants were thus placed perfectly guided. 

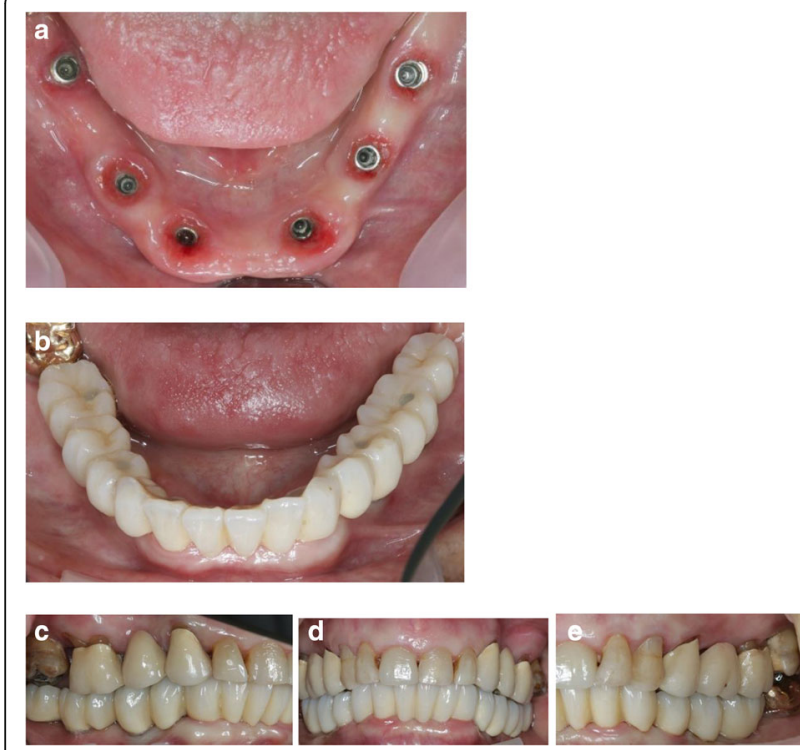

Fig. 14 Occlusal, frontal and lateral views at the day of delivery showing healthy peri-implant mucosal conditions (a) and the final CAD/CAM reconstruction in situ (b-e)

Different factors contributed to this insertion torque such as the depth of the planned implant position in a more apical area than the extraction site, the minimally invasive tooth extraction, the macroscopic implant geometry and the osteotomy protocol with a smaller drilling diameter compared to the implant diameter (as proposed by the company), the accurate performance of the single steps in the pre- and intraoperative phases, and the bone density in the anterior mandibular area. The prefabricated provisional FDP was prepared to connect the abutments to the FDP intraorally, which was easily to be performed given the accurate result of the implant positions. With this approach the passive fit of the FDP was maximized, the clinical chairside efforts (in terms of abutment connection and occlusal adaptations) were minimal and the predictability was very high compared to different limitations and problems reported in a recent review [32].

The preoperative communication between the dentist and the technician during the decision making and planning phase were essential for the concise timing in the clinic, ensuring highest surgical and prosthodontic

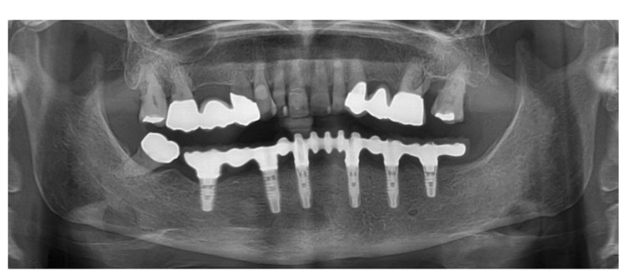

Fig. 15 Panoramic radiograph at delivery of the final CAD/CAM FDP

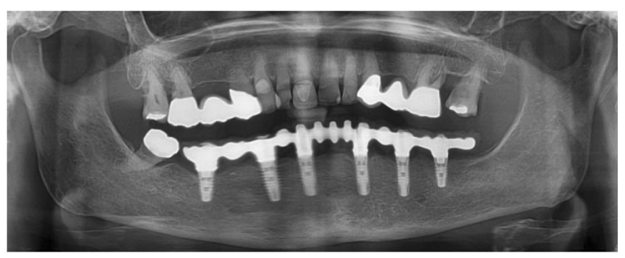

Fig. 16 Panoramic radiograph at the 12-months recall appointment

performance accuracy in this particular case. Therefore, up-to-date software and hardware with the knowledge to apply information to the specific products was required. This case report supports the need for minimally traumatic or flapless surgery, optimal implant positioning and immediate loading, as summarized in a recent review on randomized controlled trials [33].

\section{Conclusions}

The present case report emphasized the efficient workflow and the predictable outcome using computer assisted implantology. The fabrication of an immediate provisional FDP and, subsequently, the final CAD/CAM reconstruction was facilitated by CAI fulfilling patient's wish of being continuously restored during the complete treatment.

\section{Abbreviations}

CAD/CAM: Computer assisted design / computer assisted manufacturing:

CAl: Computer assisted implantology; CBCT: Cone beam computed tomography; CT: Computerized tomography; FDI: Fédération Dentaire Internationale; FDP: Fixed dental prosthesis; RPD: Removable partial denture

\section{Acknowledgements}

The authors declare no conflict of interest related to this case report.

Thommen Medical AG is acknowledged for its logistical and administrative help during the treatment.

\section{Funding}

No funding body was involved in the design of the study and collection, analysis, and interpretation of data and in writing the manuscript.

Availability of data and materials

Not applicable

\section{Authors' contributions}

TS has made substantial contributions to conception, acquisition of data, and interpretation of data. He has been involved in drafting the manuscript. He has given final approval of the version to be published and agreed to be accountable for all aspects of the work in ensuring that questions related to the accuracy or integrity of any part of the work are appropriately investigated and resolved. UH has made substantial contributions to conception, acquisition of data, and interpretation of data. He has been involved in revising the manuscript critically for important intellectual content. He has given final approval of the version to be published and agreed to be accountable for all aspects of the work in ensuring that questions related to the accuracy or integrity of any part of the work are appropriately investigated and resolved. JK has made substantial contributions to analysis and interpretation of data. He has been involved in drafting the manuscript or revising it critically for important intellectual content. He has been involved in revising the manuscript critically for important intellectual content. He has given final approval of the version to be published and agreed to be accountable for all aspects of the work in ensuring that questions related to the accuracy or integrity of any part of the work are appropriately investigated and resolved. 


\section{Ethics approval and consent to participate}

Not applicable

\section{Consent for publication}

The patient in this case report has given written informed consent for publication.

\section{Competing interests}

The authors declare that they have no competing interests.

\section{Publisher's Note}

Springer Nature remains neutral with regard to jurisdictional claims in published maps and institutional affiliations.

\section{Author details \\ ${ }^{1}$ Implantology, Oral Surgery; Private dental office, Johannesstrasse 7-9, 474623 Kevelaer, Germany. ${ }^{2}$ Department of Postgraduate Education, Faculty of Oral and Dental Medicine at J.W, Goethe-University Frankfurt am Main, Frankfurt am Main, Germany. ${ }^{3}$ Department of Reconstructive Dentistry and Gerodontology, School of Dental Medicine, University of Bern, Bern, Switzerland.}

\section{Received: 7 January 2019 Accepted: 26 March 2019}

\section{Published online: 11 April 2019}

\section{References}

1. Verstreken K, Van Cleynenbreugel J, Martens K, Marchal G, van Steenberghe $D$, Suetens P. An image-guided planning system for endosseous oral implants. IEEE Trans Med Imaging. 1998;17(5):842-52.

2. Verstreken K, Van Cleynenbreugel J, Marchal G, Naert I, Suetens P, van Steenberghe D. Computer-assisted planning of oral implant surgery: a three-dimensional approach. Int J Oral Maxillofac Implants. 1996;11(6): 806-10

3. van Steenberghe D, Naert I, Andersson M, Brajnovic I, Van Cleynenbreugel J, Suetens P. A custom template and definitive prosthesis allowing immediate implant loading in the maxilla: a clinical report. Int J Oral Maxillofac Implants. 2002;17(5):663-70.

4. Tardieu PB, Vrielinck L, Escolano E. Computer-assisted implant placement. A case report: treatment of the mandible. Int J Oral Maxillofac Implants. 2003; 18(4):599-604.

5. Avrampou M, Mericske-Stern R, Blatz MB, Katsoulis J. Virtual implant planning in the edentulous maxilla: criteria for decision making of prosthesis design. Clin Oral Implants Res. 2012;24(Suppl A100):152-9. https://doi.org/10.1111/j.1600-0501.2011.02407.x. Epub 2012 Feb 13.

6. Katsoulis J, Pazera P, Mericske-Stern R. Prosthetically driven, computerguided implant planning for the edentulous maxilla: a model study. Clin Implant Dent Relat Res. 2009:11(3):238-45.

7. Vercruyssen M, Fortin T, Widmann G, Jacobs R, Quirynen M. Different techniques of static/dynamic guided implant surgery: modalities and indications. Periodontology 2000. 2014;66(1):214-27.

8. Vasak C, Watzak G, Gahleitner A, Strbac G, Schemper M, Zechner W. Computed tomography-based evaluation of template (NobelGuide)-guided implant positions: a prospective radiological study. Clin Oral Implants Res. 2011:22(10):1157-63.

9. Van Assche N, van Steenberghe D, Guerrero ME, Hirsch E, Schutyser F, Quirynen $\mathrm{M}$, et al. Accuracy of implant placement based on pre-surgical planning of three-dimensional cone-beam images: a pilot study. J Clin Periodontol. 2007:34(9):816-21.

10. Cassetta M, Stefanelli LV, Giansanti M, Calasso S. Accuracy of implant placement with a stereolithographic surgical template. Int J Oral Maxillofac Implants. 2012;27(3):655-63.

11. Van Assche N, Quirynen M. Tolerance within a surgical guide. Clin Oral Implants Res. 2010;21(4):455-8.

12. Cassetta M, Di Mambro A, Giansanti M, Stefanelli LV, Cavallini C. The intrinsic error of a stereolithographic surgical template in implant guided surgery. Int J Oral Maxillofac Surg. 2013;42(2):264-75.

13. Cassetta M, Di Mambro A, Giansanti M, Stefanelli LV, Barbato E. How does an error in positioning the template affect the accuracy of implants inserted using a single fixed mucosa-supported stereolithographic surgical guide? Int J Oral Maxillofac Surg. 2014;43(1):85-92.
14. Schneider $D$, Marquardt $P$, Zwahlen $M$, Jung RE. A systematic review on the accuracy and the clinical outcome of computer-guided template-based implant dentistry. Clin Oral Implants Res. 2009;20(Suppl 4):73-86.

15. D'Haese J, Ackhurst J, Wismeijer D, De Bruyn H, Tahmaseb A. Current state of the art of computer-guided implant surgery. Periodontology 2000. 2017; 73(1):121-33.

16. Di Giacomo GA, da Silva JV, da Silva AM, Paschoal GH, Cury PR, Szarf G. Accuracy and complications of computer-designed selective laser sintering surgical guides for flapless dental implant placement and immediate definitive prosthesis installation. J Periodontol. 2012;83(4):410-9.

17. Tallarico M, Esposito M, Xhanari E, Caneva M, Meloni SM. Computer-quided vs freehand placement of immediately loaded dental implants: 5-year postloading results of a randomised controlled trial. Eur J Oral Implantol. 2018;11(2):203-13.

18. Tallarico M, Meloni SM. Retrospective analysis on survival rate, templaterelated complications, and prevalence of Peri-implantitis of 694 anodized implants placed using computer-guided surgery: results between 1 and 10 years of follow-up. Int J Oral Maxillofac Implants. 2017;32(5):1162-71.

19. Tallarico M, Kim YJ, Cocchi F, Martinolli M, Meloni SM. Accuracy of newly developed sleeve-designed templates for insertion of dental implants: a prospective multicenters clinical trial. Clin Implant Dent Relat Res. 2019; 21(1):108-13.

20. Zhou W, Liu Z, Song L, Kuo CL, Shafer DM. Clinical factors affecting the accuracy of guided implant surgery-a systematic review and meta-analysis. Evid Based Dent Pract. 2018;18(1):28-40

21. Seo C, Juodzbalys G. Accuracy of guided surgery via stereolithographic mucosa-supported surgical guide in implant surgery for edentulous patient: a systematic review. J Oral Maxillofac Res. 2018;9(1):e1.

22. Nkenke E, Eitner S, Radespiel-Troger M, Vairaktaris E, Neukam FW, Fenner M. Patient-centred outcomes comparing transmucosal implant placement with an open approach in the maxilla: a prospective, non-randomized pilot study. Clin Oral Implants Res. 2007;18(2):197-203.

23. Fortin $\mathrm{T}$, Bosson JL, Isidori $\mathrm{M}$, Blanchet $\mathrm{E}$. Effect of flapless surgery on pain experienced in implant placement using an image-guided system. Int J Oral Maxillofac Implants. 2006;21(2):298-304

24. Samet N, Jotkowitz A. Classification and prognosis evaluation of individua teeth--a comprehensive approach. Quintessence Int. 2009:40(5):377-87.

25. Meloni SM, Tallarico M, Pisano M, Xhanari E, Canullo L. Immediate loading of fixed complete denture prosthesis supported by 4-8 implants placed using guided surgery: a 5-year prospective study on 66 patients with 356 implants. Clin Implant Dent Relat Res. 2017;19(1):195-206.

26. Pozzi A, Holst S, Fabbri G, Tallarico M. Clinical reliability of CAD/CAM crossarch zirconia bridges on immediately loaded implants placed with computer-assisted/template-guided surgery: a retrospective study with a follow-up between 3 and 5 years. Clin Implant Dent Relat Res. 2015; 17(Suppl 1):e86-96.

27. Sigcho Lopez DA, Garcia I, Da Silva SG, Cruz LD. Potential deviation factors affecting stereolithographic surgical guides: a systematic review. Implant Dent. 2019;28(1):68-73

28. Bover-Ramos F, Vina-Almunia J, Cervera-Ballester J, Penarrocha-Diago M, Garcia-Mira B. Accuracy of implant placement with computer-guided surgery: a systematic review and meta-analysis comparing cadaver, clinical, and in vitro studies. Int J Oral Maxillofac Implants. 2018;33(1):101-15.

29. Marliere DAA, Demetrio MS, Picinini LS, De Oliveira RG, Chaves Netto HDM. Accuracy of computer-guided surgery for dental implant placement in fully edentulous patients: a systematic review. Eur J Dent. 2018;12(1):153-60.

30. Pozzi A, Polizzi G, Moy PK. Guided surgery with tooth-supported templates for single missing teeth: a critical review. Eur J Oral Implantol. 2016:9(Suppl 1):S135-53.

31. Raico Gallardo YN, da Silva-Olivio IRT, Mukai E, Morimoto S, Sesma N, Cordaro L. Accuracy comparison of guided surgery for dental implants according to the tissue of support: a systematic review and meta-analysis. Clin Oral Implants Res. 2017;28(5):602-12.

32. Tahmaseb A, Wismeijer D, Coucke W, Derksen W. Computer technology applications in surgical implant dentistry: a systematic review. Int J Oral Maxillofac Implants. 2014;29(Suppl):25-42.

33. Colombo M, Mangano C, Mijiritsky E, Krebs M, Hauschild U, Fortin T. Clinical applications and effectiveness of guided implant surgery: a critical review based on randomized controlled trials. BMC Oral Health. 2017;17(1):150. 